Cita bibliográfica: Cardoso, L., Araújo Vila, N., de Almeida, A. y Fraiz Brea, J. A. (2019). Los festivales de música como inductores de imagen de destino turístico. El caso del Festival Vodafone Paredes de Coura. Investigaciones Turísticas (17), pp. 149-167. http://dx.doi.org/10.14198/INTURI2019.17.07

\title{
Los festivales de música como inductores de imagen de destino turístico. El caso del Festival Vodafone Paredes de Coura
}

\section{Music festivals as promoters of tourism destination image. The case of the Vodafone Paredes de Coura Festival}

Lucília Cardoso, Universidad de Vigo, España. lucyalves.lucilia@gmail.com

Noelia Araújo Vila, Universidad de Vigo, España. naraujo@uvigo.es

Ângela de Almeida, ESACT-IP Bragança, Portugal. acma.mdl@gmail.com

José Antonio Fraiz Brea, Universidad de Vigo, España. jafraiz@uvigo.es

\section{RESUMEN}

Turismo y eventos no son un fenómeno reciente, lo que es novedoso es la importancia y la influencia de los eventos en la imagen del destino. Los eventos estimulan el desarrollo de los destinos, son instrumentos de construcción de imagen de un territorio, aumentan la visibilidad turística y complementan la promoción turística. En la oferta de eventos de tipo turístico, destacan los festivales de música y entre ellos el Festival Vodafone de Paredes de Coura (con más de 100.000 asistentes), que se celebra en la villa de Paredes de Coura en el destino turístico Porto y Norte (Portugal). La presente comunicación tiene como objetivo principal verificar si la imagen del festival afecta positivamente a la imagen del destino turístico. Para ello, se ha diseñado una encuesta y se ha recogido de modo aleatorio a 395 asistentes al festival, en la que se valoran un total de 24 ítems sobre la imagen del festival y del destino y se analiza el perfil del festivalero.

Palabras clave: imagen de destino, evento, festival de música, imagen cognitiva.

\section{ABSTRACT}

Tourism and events are not a recent phenomenon. What is new and important is the influence that events have on a destination's image. Events stimulate the development of destinations, they are image-building instruments of a territory, they increase the visibility of destinations and they complement tourism promotion. In the portfolio of events as tourist products, we can highlight music festivals, including the Vodafone Festival of Paredes de Coura (with more than 100,000 attendees), which takes place in the town of Paredes de Coura in the tourist destination of Porto-North (Portugal). The main objective of this article is to determine whether the image of the festival positively affects the image of the tourist destination. For this purpose, a 
questionnaire has been randomly administered among 395 individuals attending the festival, in which a total of 24 items of the festival and destination image are evaluated. The profile of the festival is also analyzed.

Keywords: destination image, event, music festival, cognitive image.

\section{INTRODUCCIÓN}

El estudio de la imagen empezó a recibir más atención cuando se verificó que ésta era una de las principales responsables para la elección de un destino turístico y que estaba vinculada a la satisfacción y a la intención de revisitarlo (Añaña et al., 2016). Además, es un factor importante a considerar cuando se desarrollan estrategias de marketing para el destino (Baloglu y McCleary, 1999). Así, con la creciente variedad y la aparición de destinos turísticos que presionan ante la necesidad de diferenciación a través de estrategias de posicionamiento y comunicación, se hace vital entender el Tourism Destination Image (TDI) (Brea y Cardoso, 2011) de forma que haga frente a los competidores con una oferta turística a la altura (Cardoso y Brea, 2012; Hosany et al., 2007). La imagen del destino turístico surge como impulsora de la competitividad de los destinos (Stepchenkova y Li, 2014), donde destinos con imagen más fuerte y positiva tienen más probabilidad de ser escogidos por el turista (Beerli y Martín, 2004; Echtner y Ritchie, 2003), lo que refuerza la necesidad del branding y del brand image (Cardoso y Marques, 2015).

El brand image -que agrupa "atributos, actitudes y beneficios" (Stepchenkova y Li, 2014)-, contribuye a la construcción de la identidad del lugar. Por tanto, el brand image desempeña un papel de éxito en los destinos turísticos (Beerli y Martín, 2004). Comentar también que en la perspectiva del turismo y de las Destination Marketing Organizations (DMO), los festivales/eventos son vistos como image-makers (Getz, 2008) porque cuando se vinculan a la estrategia de comunicación del territorio pueden tener una función promocional turística, pueden realzar la identidad territorial y "fabricar" y consolidar la marca del destino (Morales y Vela, 2009). Además, al mejorar la imagen del destino, promueven la comercialización de productos turísticos, el compartir la cultura y el crear actividades de ocio para los residentes reforzando la experiencia turística al permitir compartirla entre el turista y el residente (Zucco de Quadros et al., 2017). Por tanto, la imagen del destino es un estímulo y motivación de viaje para el turista. Por ello, la promoción turística no debe descuidar la inclusión de los eventos ligados a la identidad y características únicas del lugar (Paiva, 2015; Getz y Page, 2016; Zucco et al., 2017).

La imagen de un destino turístico depende de las percepciones y de los contenidos generados por viajeros y residentes (Llodrá-Riera et al., 2015). Por ello, el presente trabajo se centra precisamente en la percepción de uno de estos dos colectivos, los viajeros, y, concretamente, en los viajeros festivaleros que se desplazan motivados por un evento, en este caso un evento musical. Para ello, se realiza en primer lugar una contextualización teórica centrada en la TDI y los festivales de música dentro de los eventos inductores de imagen turística de un destino. Como parte práctica del estudio se recoge una muestra aleatoria de 395 encuestas a los asistentes a un festival, el Festival Vodafone Paredes de Coura (FVPC) situado en Viana do Castelo, en la región de Turismo de Porto y Norte de Portugal. Este festival fue creado por un 
grupo de amigos locales en 1993, festival que vendría a colocar a Paredes de Coura en el mapa como "meca del indi". Se celebra habitualmente en el mes de agosto en un anfiteatro natural único, proporcionando a los festivaleros el contacto con la naturaleza y música de una forma harmoniosa. Paredes de Coura es una villa situada en el interior del alto Minho que debido a la celebración del festival ha ganado fama. Los agentes locales son los mayores impulsores involucrándose en la organización del evento con entusiasmo, ya que los días del festival son parte importante en la economía local. El objetivo general es verificar si existe relación positiva entre la imagen cognitiva del Festival Vodafone de Paredes de Coura y el destino turístico, así como analizar el perfil de asistente a este festival.

\section{TOURISM DESTINATION IMAGE (TDI) Y SUS ESPECIFICIDADES}

El estudio de la imagen del destino turístico tiene su inicio en los años setenta y muestra una infinidad de conceptos dependiendo del área científica, del investigador o del interés de la investigación. Según Gallarza, Saura y García (2002, p. 68) "existen tantas definiciones como autores interesados en conceptualizarla". Analizando el concepto más recientemente, la falta de consenso relativo a la definición persiste (Hunter, 2016; Pike, 2016), pero, existe acuerdo en considerar que la TDI está constituida por componentes cognitivos y afectivos que están jerárquicamente relacionados y forman una impresión global del destino turístico (Agapito et al., 2013; Brea y Cardoso, 2011; Josiassen, et al., 2016; Mak, 2017; Martín-Santana et al., 2017). El componente cognitivo, también designado perceptual o intelectual, se define como "la suma de creencias y actitudes hacia un objeto que conduce a algunas imágenes internas de sus atributos" (Gartner, 1993, p. 93). Es una dimensión de la interpretación de las características del destino (Loureiro y Araujo, 2015) que refleja las percepciones de los turistas relativas a los atributos del destino o, en definitiva, es lo que piensa un individuo o piensa que sabe del destino (Önder y Marchiori, 2017). El componente afectivo es una dimensión fruto de las percepciones positivas o negativas que el turista tiene en relación al lugar (Loureiro y Araujo, 2015) representada por las respuestas emocionales (Stylos et al., 2017). Abarca las emociones (Hallmann et al., 2015) y los sentimientos relacionados con el destino y es crucial en el diálogo del turista con el destino y en la creación de lealtad (Agapito et al., 2013).

El resultado de la suma de los componentes cognitivo y afectivo es la imagen global, también designada componente conativo, un componente de acción (Gartner, 1993) que abarca la forma de actuar y permanece hasta que los turistas hacen sus elecciones (Pan y Li, 2011; Stepchenkova y Morrison, 2008). Además, según Beerli y Martín (2004), la imagen del destino turístico es un cuadro mental formado por un conjunto de atributos que definen el destino en sus diversas dimensiones. En este contexto, el componente cognitivo de la imagen del destino es el proceso mental por el cual los turistas evocan en la memoria los atributos del mismo (Cardoso y Brea, 2012).

En el contexto de los eventos, y en lo que se refiere a la TDI, Custódio y Gouveia (2004) presentan el componente cognitivo como la evaluación de los atributos del evento, siendo positivos o negativos. En el contexto de la imagen del destino turístico, Beerli y Martín (2004) defienden que este componente está basado en las evaluaciones de los atributos del destino. Continuando la argumentación, existe todavía unanimidad en la literatura en considerar a los 
festivales, en su vertiente de festivales de música, potenciadores de la inducción de la imagen de un destino turístico porque los festivales no se limitan al consumo de este producto turístico específico, sino que consumen el destino en su globalidad (Custódio y Gouveia, 2004). Por tanto, comprender las evaluaciones que los festivaleros hacen de los atributos de un destino durante el transcurso de un festival de música es crucial para los gestores de dicho lugar.

\section{LOS EVENTOS COMO INDUCTORES DE IMAGEN DE DESTINO TURÍSTICO: LOS FESTIVALES DE MÚSICA}

La creación y promoción de eventos es una de las estrategias seguidas por las DMOs para incrementar el atractivo del destino y atraer a un mayor número de turistas y visitantes. Estos se encuadran dentro del denominado turismo cultural y dan respuesta a las exigencias de los turistas vigentes desde inicio de este siglo: novedad, socialización, prestigio, descanso, enriquecimiento intelectual (Araújo y Domínguez, 2012); y sobre todo se puede considerar un tipo de turismo alternativo incluido en el tan en boga turismo experiencial (Estrada et al., 2015).

Los eventos aumentan la visibilidad de un destino turístico (Paiva, 2015), refuerzan su atractivo, auxilian en la promoción (Getz y Page, 2016) y superan los efectos de la estacionalidad del destino turístico (Weaver y Lawton, 2013). También suponen un impulso para el desarrollo y consumo del turismo cultural dentro de la reconocida economía de experiencias, mejoran la imagen de la ciudad y facilitan la regeneración urbana (Liu, 2014). Es por ello que, el sector turístico debería esforzarse en ofrecer experiencias inolvidables a través de diversos eventos y no sólo durante el momento del disfrute sino en el post-consumo (Hosany y Witman, 2010).

Portugal reconoce la importancia y el potencial de los eventos para promover y dar visibilidad al destino, y así está contemplado en la estrategia de turismo del país. En la estrategia de Turismo 2027 (de Portugal) los eventos se encuentran asociados a temas como: la proyección y el aumento de notoriedad de Portugal en los mercados internacionales, el aumento de la búsqueda de atractivo, el estímulo de las economías locales, y, además, aumentar el valor del territorio y enriquecer la experiencia turística. Los eventos culturales muestran una clara ambición en proyectar la imagen del destino turístico portugués a nivel internacional, con oferta a nivel de congresos, eventos y deporte. Los eventos son también parte de la estrategia de posicionamiento del destino interno y se usan para la promoción turística de los territorios (Turismo de Portugal I.P., 2017).

Dentro de los eventos culturales, uno de los destacados de la cartera de productos turísticos de un destino son los festivales (Getz, 2008). Los festivales son definidos como "eventos que consisten en una serie de actuaciones y programas, muchas veces dedicados a un único género, cargado de alegría y cordialidad" (Yan et al., 2017), o como "la celebración de un tema específico al cual el público es invitado por un periodo limitado de tiempo. Esta celebración puede ser anual o con menor frecuencia, e incluso eventos de carácter único" (Grappi y Montanari, 2011, p. 1129). Son reconocidos como estrategia de acogida de un destino, vía para obtener beneficios, y no sólo económicos, sino sociales y culturales (Grappi y Montanari, 2011) y son un medio de promoción de los destinos turísticos (Morales y Vela, 2009). Además, 
los festivales locales, son un buen mecanismo para impulsar y dar a conocer costumbres culturales únicas (Lee et al., 2011).

Los festivales no suponen solo el consumo de un tipo concreto de producto, sino que se crea un espacio en el que se dan relaciones culturales, humanas y sociales (Prat, 2014). El principal beneficio es por tanto la propia experiencia vivida (Seonjeong et. al, 2014).

Están también vinculados a la difusión de la cultura (Zucco et al., 2017) y son elementos esenciales en los planes de marketing del destino (Getz y Page, 2016; Tanford y Jung, 2017). Los festivales están creciendo rápidamente en la industria del turismo debido a su potencial de desarrollo del territorio en términos de reposicionamiento del destino, revitalización y reestructuración económica (Pavlukovic et al., 2017), pero hace solamente unas décadas que el turismo de eventos empezó a destacar y tener relevancia en las investigaciones y desde entonces su importancia y estudio han aumentado considerablemente (Getz y Page, 2016). Éstas han seguido varias direcciones, como la relación de los eventos con la imagen del destino turístico y su potencial en image-enhancement del destino, es decir, como los eventos pueden mejorar y perfeccionar la imagen del destino (Getz, 2008), donde las propias autoridades del destino usan los eventos para la construcción de la imagen, reputación e identidad (Santos et al., 2017).

Además, pensando en un largo plazo, variables como el precio y los esfuerzos de venta online deben tenerse en cuenta y trabajarse. De su buen uso y éxito depende la salud en el tiempo de un festival. La estrategia de marca a largo plazo es también vital. Características de la marca como la imagen, el ambiente y las emociones atraen a las personas, las hacen leales y los visitantes pueden gastar más dinero en eventos de alta equidad de marca (Leenders, 2010). Resultados de la investigación llevada a cabo por Llopis et al. (2017) permiten confirmar el impacto positivo y significativo de la comunicación controlada por la organización de un evento sobre la notoriedad de marca. Se concluye también que la percepción del valor de marca global del festival por parte del asistente tiene un efecto positivo y significativo en su satisfacción y en sus intenciones conductuales.

Al hablar de festivales pensamos en música, cine, arte, danza o teatro, entre los principales. En Portugal se han celebrado en 2016246 festivales de música (un 18\% más que el año anterior, con 210) según la APORFEST - Associação Portuguesa Festivais de Música-, destacando así entre los demás. El número de espectadores se cifró en 2,1 millones (equivalente a un quinto de la población portuguesa). Los festivales de música portuguesa son un sector en crecimiento desde 2012, tanto en número de festivales como en número de asistentes, aunque presentan una serie de debilidades que deben mejorar. Una de ellas es la continuidad en el tiempo (algunos no superan la segunda, tercera o cuarta edición), sobreviviendo solo aquellos considerados "grandes festivales", por contar con apoyo y patrocinios (APORFEST, 2017).

Entre los principales de 2016 se encuentran Rock in Río Lisboa, Nos em D’abandada y Meo Sudoeste, superando prácticamente los 200.000 asistentes (Tabla 1). En quinta posición, con 100.000 espectadores, se encuentra el Festival de Paredes de Coura (en el que se han recogido los datos de la parte empírica del trabajo). 
Tabla 1. Ranking festivales de música Portugal 2016. Top Five

\begin{tabular}{|c|c|c|c|c|}
\hline$\#$ & Festival & No espectadores & № días & Entrada \\
\hline 1 & Rock in Río & 329.000 & 5 & PAGA \\
\hline 2 & Nos em D’abandada & 200.000 & 1 & GRATIS \\
\hline 3 & Meo Sudoeste & 195.000 & 5 & PAGA \\
\hline 4 & Nos Alive & 165.000 & 3 & PAGA \\
\hline 5 & RFM Somnii & 100.000 & 3 & PAGA \\
\hline 5 & Vodafone Paredes de Coura & 100.000 & 4 & PAGA \\
\hline 5 & Festival do Crato & 100.000 & 4 & GRATIS/PAGA \\
\hline 5 & FMM Sines & 100.000 & 10 & \\
\hline
\end{tabular}

Fuente: APORFEST (2017)

Las grandes citas musicales atraen a un turista de diverso gusto musical que tiene como característica común una alta predisposición a gastarse una elevada cantidad de dinero en los lugares que visita (Chierichetti, 2012). Por todo ello, es más que evidente la importancia que éstos tienen como atractivo turístico, debiendo incluirse en la estrategia promocional de un destino y ser considerados como inductores de imagen turística.

\section{EL FESTIVAL VODAFONE PAREDES DE COURA Y EL DESTINO TURÍSTICO DE VIANA DO CASTELO}

Viana do Castelo forma parte de la región de turismo Porto y Norte. Los productos estratégicos ofertados por esta entidad regional se dividen en siete categorías: turismo de negocios; city and short breaks; gastronomía y vinos; turismo de naturaleza; turismo religioso; turismo cultural y paisajístico; y salud y bienestar.

Viana do Castelo pertenece también a la región del Minho y debido a las especificidades de sus recursos y productos turísticos sus mercados objetivo son el segmento de naturaleza (turismo activo) y el segmento de turismo cultural y paisajístico.

A nivel cultural la web www.portoenorte.pt/pt/touring-cultural-e-paisagistico menciona los festivales de música de la región. Realza que la región fue pionera en realizar un festival de música al aire libre en Portugal en el año 1965, el Festival de Vilar de Mouros. En cuanto al Festival de "Paredes de Coura", afirma que se celebra en uno de los sitios más improbables del interior, pero de elevada belleza y un anfiteatro natural, el de la playa fluvial de Taboão. También hace alusión al Milhões da Festa en Barcelos y al Primavera Sound en Porto. Además, la región cuenta con otros eventos de gran importancia y que no están mencionados en la web, como el "Neopop", un festival más reciente que se celebra también en Viana do Castelo.

Centrándonos en el festival objeto de estudio, éste fue creado por un grupo de amigos locales en 1993, cuenta ya con la 26a edición y sitúa a Paredes de Coura en el mapa como la "meca del indie". Se enmarca en un anfiteatro natural único, proporcionando a las actuaciones una mezcla de contacto con la naturaleza y música de carácter único. Paredes de Coura es una 
villa del interior del alto Minho que debido a la realización del festival se hizo famosa, ya que los agentes locales son los mayores impulsores involucrados en la organización del evento con todo el entusiasmo, pues, estos días de festival son parte importante en la economía local. Los residentes locales ofrecen sopa y café en casa, los jardines de sus casas para acampar y algunas mangueras para bañarse, ingredientes que marcan la magia del festival.

Es el festival más antiguo y carismático del país, conocido por su espíritu alternativo y el eslogan publicitado en su sitio web, "El hábitat natural de la música", hace justicia al evento en el que se suelen dar a conocer nuevos nombres de la música nacional y mundial (Figura 1).

Figura 1. Website del Festival Vodafone Paredes de Coura

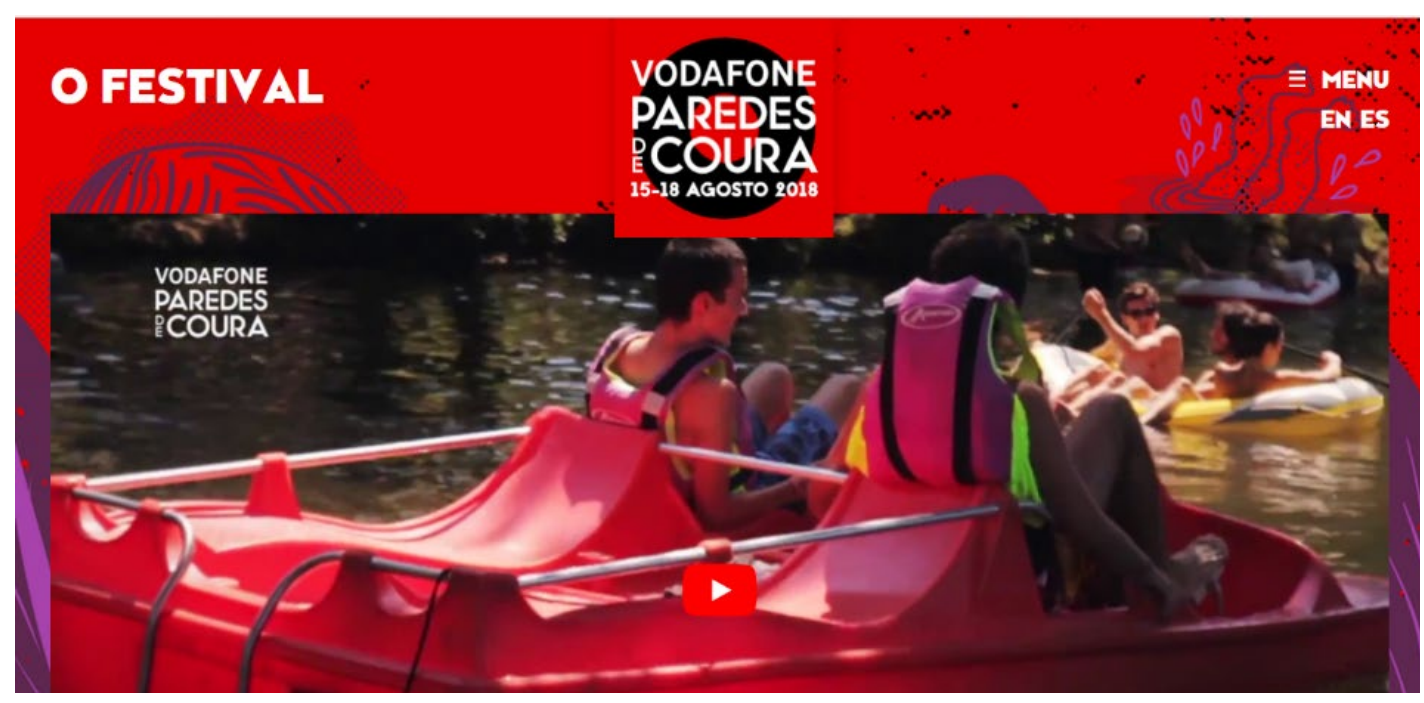

Fuente: www.paredesdecoura.com

La organización del festival percibió que a pesar de la notoriedad que éste tiene es necesario promover y comunicar el evento, siendo aquí de donde surge el actual gran patrocinador que lo respalda (Vodafone). Es también imprescindible mencionar que el Festival Vodafone Paredes de Coura tiene una marca sólida en los nombres de festivales de verano nacionales e internacionales, pero es consciente de la importancia de la marca del principal patrocinador, Vodafone, como herramienta para añadir valor positivo en la comunicación al público.

En realidad, como se puede observar in situ, la marca Vodafone está ubicada en puntos estratégicos y respeta a los diversos públicos de este festival a la hora de la distribución de los regalos, entre conciertos, en la zona de acampada y en las diversas actividades que lo componen, pues es un festival donde hay jazz en el césped, recitación de poesía y comedia. Así, de esta forma, los festivaleros pueden apreciar todos los momentos sin la "contaminación acústica" existente en otros eventos con patrocinadores. 


\section{OBJETIVO Y METODOLOGÍA}

El objetivo del presente trabajo se resume en la siguiente cuestión: ¿existe relación positiva entre la imagen del Festival Vodafone Paredes de Coura y la imagen del destino turístico de Viana do Castelo? Para responder a ello, se presenta la siguiente hipótesis: la imagen cognitiva del Festival Vodafone Paredes de Coura (ICFVPC) afecta positivamente a la imagen cognitiva del destino turístico de Viana do Castelo (ICDTVC).

Para responder al objetivo principal se plantean dos sub-objetivos secundarios: (1) trazar el perfil del festivalero del FVPC incluyendo el comportamiento del viaje; (2) mostrar la imagen cognitiva del FVPC y del destino turístico de Viana do Castelo. Como método de investigación se ha optado por la recogida de datos primarios a través de la técnica cuantitativa del cuestionario, estructurado con constructos de escala Likert de 5 puntos, tanto para el ICFVPC como el ICDTVC, siendo el muestreo elegido el aleatorio. Para acceder a los atributos del FVPC se ha seguido el modelo de Custódio y Gouveia (2007) y para los atributos del destino de Viana do Castelo se ha seguido el modelo de Beerli y Martín (2004).

El número de encuestas recogidas fue de 395 , que, en una población de carácter infinito, representa un error muestral del $\pm 4,9 \%$ (para un nivel de confianza del $95 \%$ ). La recogida de los datos fue a lo largo de los cuatro días de celebración del festival (del 17 al 20 de agosto de 2016) en los espacios del propio festival, principalmente los márgenes de la playa fluvial de Tabuão, donde se celebraron los conciertos de jazz, en las zonas de ocio, en la zona de acampada y en el recinto de los conciertos.

Para el tratamiento de los datos se ha recurrido a la metodología cuantitativa: (1) para el perfil de la muestra se ha usado la estadística descriptiva con recuento de frecuencias (relativas y absolutas); (2) el comportamiento del viaje se ha analizado mediante estadística descriptiva con un recuento de frecuencias (relativas y absolutas); mínimo y máximo, promedio y desviación típica; (3) para la estructura de la imagen cognitiva de Viana do Castelo y FVPC se ha usado la estadística descriptiva - media y desviación típica; (4) para la relación entre el Festival Vodafone Paredes de Coura y el destino Viana do Castelo se ha usado el análisis inferencial de Correlación - coeficiente de correlación de Spearman.

\section{RESULTADOS}

\section{Perfil del visitante del FVPC}

En primer lugar, se analiza el perfil de la muestra, reflejado en la Tabla 2. Dicha información permite verificar que la variable género está equilibrada, mientras que la franja de edad más habitual es la que va de 18 a 23 años (52,15\% de la muestra), seguida de 24 a 29 años $(23,54 \%)$. 
Tabla 2. Perfil de la muestra

\begin{tabular}{|c|c|c|}
\hline \multirow[t]{2}{*}{ Variables demográficas } & Muestra, $n=395$ & \\
\hline & Núm. de casos & Porcentaje\% \\
\hline \multicolumn{3}{|l|}{ Género } \\
\hline Masculino & 216 & $54,7 \%$ \\
\hline Femenino & 179 & $45,3 \%$ \\
\hline \multicolumn{3}{|l|}{ Edad } \\
\hline$<18$ años & 36 & $9,1 \%$ \\
\hline 18-23 años & 206 & $52,2 \%$ \\
\hline 24-29 años & 93 & $23,5 \%$ \\
\hline 30-39 años & 37 & $9,4 \%$ \\
\hline$>39$ años & 22 & $5,6 \%$ \\
\hline No ha respondido & 1 & $0,3 \%$ \\
\hline Mín. - Máx. & \multicolumn{2}{|c|}{ 14-74 Años } \\
\hline Media \pm Desviación & \multicolumn{2}{|c|}{$23,9 \pm 7,3$ Años } \\
\hline Q1 - Mediana - Q3 & \multicolumn{2}{|c|}{ 19-22 - 26 Años } \\
\hline \multicolumn{3}{|l|}{ Situación Profesional } \\
\hline Trabajador & 140 & $35,4 \%$ \\
\hline Desempleado & 10 & $2,5 \%$ \\
\hline Jubilado & 1 & $0,3 \%$ \\
\hline Tareas domésticas & 1 & $0,3 \%$ \\
\hline Estudiante & 220 & $55,7 \%$ \\
\hline No ha respondido & 23 & $5,8 \%$ \\
\hline \multicolumn{3}{|l|}{ Estudios } \\
\hline Educación básica & 1 & $0,3 \%$ \\
\hline Educación secundaria & 201 & $50,9 \%$ \\
\hline Licenciatura/Grado & 118 & $29,9 \%$ \\
\hline Master/Postgrado & 55 & $14,0 \%$ \\
\hline No ha respondido & 20 & $5,1 \%$ \\
\hline \multicolumn{3}{|l|}{ Nacionalidad } \\
\hline Portuguesa & 315 & $79,7 \%$ \\
\hline Extranjera & 58 & $20,3 \%$ \\
\hline Española & 40 & $10,2 \%$ \\
\hline Alemana & 7 & $1,8 \%$ \\
\hline Brasileira & 6 & $1,5 \%$ \\
\hline No ha respondido & 17 & $4,3 \%$ \\
\hline \multicolumn{3}{|l|}{ País de Residencia } \\
\hline Portugal & 327 & $82,8 \%$ \\
\hline España & 41 & $10,4 \%$ \\
\hline Alemania & 5 & $1,3 \%$ \\
\hline Austria & 4 & $1,0 \%$ \\
\hline Francia & 4 & $1,0 \%$ \\
\hline Reino Unido & 4 & $1,0 \%$ \\
\hline Otros países & 10 & $2,5 \%$ \\
\hline \multicolumn{3}{|l|}{ Distrito de Residencia de los portugueses } \\
\hline Porto & 94 & $28,8 \%$ \\
\hline Lisboa & 75 & $23,0 \%$ \\
\hline Braga & 32 & $9,8 \%$ \\
\hline Viana do Castelo & 28 & $8,6 \%$ \\
\hline Aveiro & 26 & $8,0 \%$ \\
\hline Coímbra & 17 & $5,2 \%$ \\
\hline \multicolumn{3}{|l|}{ Motivación del viaje } \\
\hline Viaja en vacaciones & 17 & $4,3 \%$ \\
\hline Viaja para asistir al festival & 279 & $70,6 \%$ \\
\hline Viaja en vacaciones y asiste al festival & 98 & $24,8 \%$ \\
\hline No ha respondido & 1 & $0,3 \%$ \\
\hline
\end{tabular}


En cuanto a la profesión, el asistente más habitual es estudiante (57,6\% de la muestra), y el nivel educativo mayoritario es la educación secundaria (50,9\%). La mayoría de los asistentes son de nacionalidad portuguesa (82,5\%), desplazándose desde Porto, Lisboa, Braga, Aveiro y Coimbra. Tan solo el $8,6 \%$ son residentes del mismo lugar de celebración del festival (Viana do Castelo). Entre los extranjeros destaca la nacionalidad española (10,2\%), esperable por la ubicación del festival. Aun así, el 6,8\% de los asistentes provienen de otros países (Alemania, Austria, Francia y Reino Unido principalmente).

Un dato relevante es la motivación de viaje, ya que el 70,6\% se desplaza para asistir al festival, convirtiéndose en la motivación principal del viaje, frente a los que lo hacen porque están de vacaciones (4,3\%).

Por tanto, y dando respuesta a uno de los objetivos de este trabajo (definir el perfil de asistente al Festival Vodafone Paredes de Coura), la muestra recogida concluye con el siguiente perfil: los visitantes del Festival Vodafone Paredes de Coura son en su mayoría jóvenes estudiantes con hasta el momento enseñanza secundaria, de sexo tanto masculino como femenino, de una edad entre 18 y 23 años. La nacionalidad predominante es la portuguesa (82\%), residentes en Lisboa, siguiéndoles en mucho menor cantidad los españoles (10\%), en su caso procedentes de Madrid.

\section{Comportamiento del viaje}

Con el fin de completar y concretar este perfil, se realizó un segundo bloque de preguntas a los encuestados (Tabla 3). En lo que respecta a la compañía, la mayoría acude acompañados (92,2\%), siendo lo más habitual de este colectivo ir con los amigos (79\%) y en grupos de 4 a 5 personas (28,3\%); mientras que tan solo el $4,8 \%$ acude en solitario.

En cuanto a los días que permanece en la región, lo más habitual son 5-7 días (51,1\%), mientras que en el festival lo hace 4 (38\%). El tipo de alojamiento por excelencia es la propia zona de acampada ofertada por el festival $(81,8 \%)$. 
Tabla 3. Comportamiento del viaje de los festivaleros del FVPC

\begin{tabular}{|c|c|c|}
\hline Variables del comportamiento del viaje & Muestra, $\mathrm{n}=395$ & \\
\hline & Núm. de casos & Porcentaje\% \\
\hline \multicolumn{3}{|l|}{ Cómo viaja } \\
\hline Solo & 19 & $4,8 \%$ \\
\hline Acompañado & 364 & $92,2 \%$ \\
\hline No ha respondido & 12 & $3,0 \%$ \\
\hline \multicolumn{3}{|l|}{ De quién se acompaña } \\
\hline Amigos & 286 & $79 \%$ \\
\hline Familia & 32 & $8,1 \%$ \\
\hline Amigos y familia & 21 & $5,3 \%$ \\
\hline Pareja & 25 & $6,3 \%$ \\
\hline Otros & 5 & $1,3 \%$ \\
\hline \multicolumn{3}{|l|}{ Número total de personas que lo acompañan } \\
\hline 1 persona & 39 & $10,7 \%$ \\
\hline 2-3 personas & 79 & $21,7 \%$ \\
\hline 4-5 personas & 103 & $28,3 \%$ \\
\hline 6-10 personas & 80 & $22,0 \%$ \\
\hline 11-21 personas & 51 & $14,1 \%$ \\
\hline No ha respondido & 12 & $3,2 \%$ \\
\hline Mín.- Máx. & \multicolumn{2}{|c|}{ 1-51 personas } \\
\hline Media \pm Desviación & \multicolumn{2}{|c|}{$6,4 \pm 5,8$ personas } \\
\hline Q1 - Mediana-Q3 & \multicolumn{2}{|c|}{ 3-5 - 8 personas } \\
\hline \multicolumn{3}{|l|}{ Cuántos días permanece en la región } \\
\hline 1 día & 11 & $2,8 \%$ \\
\hline 2 días & 22 & $5,6 \%$ \\
\hline 3 días & 5 & $1,3 \%$ \\
\hline 4 días & 65 & $16,5 \%$ \\
\hline 5-7 días & 202 & $51,1 \%$ \\
\hline 8-14 días & 76 & $19,3 \%$ \\
\hline 15-30 días & 10 & $2,6 \%$ \\
\hline No ha respondido & 4 & $1,0 \%$ \\
\hline Min- Máx & \multicolumn{2}{|c|}{ 1-30 días } \\
\hline Media \pm Desviación & \multicolumn{2}{|c|}{$6,1 \pm 3,1$ días } \\
\hline Q1 - Mediana-Q3 & \multicolumn{2}{|c|}{ 4-6- 7 días } \\
\hline \multicolumn{3}{|l|}{ Cuántos días permanece en el festival } \\
\hline 1 día & 23 & $5,8 \%$ \\
\hline 2 días & 16 & $4,1 \%$ \\
\hline 3 días & 11 & $2,8 \%$ \\
\hline 4 días & 150 & $38,0 \%$ \\
\hline 5-7 días & 134 & $33,9 \%$ \\
\hline 8-15 días & 56 & $14,3 \%$ \\
\hline No ha respondido & 5 & $1,3 \%$ \\
\hline Mín.- Máx. & \multicolumn{2}{|c|}{ 1-15 días } \\
\hline Media \pm Desviación & \multicolumn{2}{|c|}{$5,0 \pm 2,1$ días } \\
\hline Q1 - Mediana-Q3 & \multicolumn{2}{|c|}{ 4-4-6 días } \\
\hline \multicolumn{3}{|l|}{ Qué tipo de alojamiento utiliza } \\
\hline Acampada del festival & 323 & $81,8 \%$ \\
\hline Alojamiento Turístico local & 16 & $4,1 \%$ \\
\hline No ha respondido & 56 & $14,2 \%$ \\
\hline
\end{tabular}




\section{Imagen cognitiva del FVPC (ICFVPC)}

La ICFVPC realizada a través de una escala Likert de 5 puntos se muestra en la Tabla 4. La evaluación cognitiva de los atributos del FVPC tiene mayor frecuencia de concordancia plena en los ítems 5 -el paisaje del festival es bonito- (77\% lo han valorado con 5 puntos), ítem 2 -los espacios del festival son seguros- (53,4\% lo valora con 5), ítem 1 -la organización del evento es adecuada- (45,1\% lo valora con 5$)$ y el ítem 6 -existe una buena recepción de los fans- $(43,4 \%$ lo valoran con 5$)$. En lo que respecta al resto de ítems, a pesar de no obtener valoraciones máximas, estos obtienen valoraciones de 4 sobre 5 .

Tabla 4. ICFVPC - atributos del Festival Vodafone Paredes de Coura

\begin{tabular}{|c|r|r|r|r|r|r|c|}
\hline & \multicolumn{7}{|c|}{ Clasificación del Evento } \\
\hline Ítems & $\mathbf{1}$ & $\mathbf{2}$ & $\mathbf{3}$ & $\mathbf{4}$ & $\mathbf{5}$ & No responde & $\begin{array}{c}\text { Media } \pm \\
\text { Desviación }\end{array}$ \\
\hline $\mathbf{1}$ & $2(0,5 \%)$ & $12(3,0 \%)$ & $36(9,1 \%)$ & $164(41,5 \%)$ & $178(45,1 \%)$ & $3(0,8 \%)$ & $4,3 \pm 0,8$ \\
\hline $\mathbf{2}$ & $3(0,8 \%)$ & $4(1,0 \%)$ & $23(5,8 \%)$ & $151(38,2 \%)$ & $211(53,4 \%)$ & $3(0,8 \%)$ & $4,4 \pm 0,7$ \\
\hline $\mathbf{3}$ & $3(0,0 \%)$ & $9(2,3 \%)$ & $68(17,2 \%)$ & $193(48,7 \%)$ & $115(29,0 \%)$ & $8(2,0 \%)$ & $4,1 \pm 0,8$ \\
\hline $\mathbf{4}$ & $10(2,5 \%)$ & $36(9,1 \%)$ & $71(17,9 \%)$ & $142(35,9 \%)$ & $128(32,3 \%)$ & $9(2,3 \%)$ & $3,9 \pm 1,1$ \\
\hline $\mathbf{5}$ & $0(0,0 \%)$ & $2(0,5 \%)$ & $13(3,3 \%)$ & $69(17,4 \%)$ & $305(77,0 \%)$ & $7(1,8 \%)$ & $4,8 \pm 0,5$ \\
\hline $\mathbf{6}$ & $4(1,0 \%)$ & $12(3,0 \%)$ & $49(12,4 \%)$ & $150(37,9 \%)$ & $172(43,4 \%)$ & $9(2,3 \%)$ & $4,2 \pm 0,9$ \\
\hline $\mathbf{7}$ & $4(1,0 \%)$ & $27(6,8 \%)$ & $83(21,0 \%)$ & $159(40,2 \%)$ & $113(28,5 \%)$ & $10(2,5 \%)$ & $3,9 \pm 0,9$ \\
\hline $\mathbf{8}$ & $23(5,8 \%)$ & $50(12,6 \%)$ & $94(23,7 \%)$ & $145(36,6 \%)$ & $79(19,9 \%)$ & $5(1,3 \%)$ & $3,5 \pm 1,1$ \\
\hline $\mathbf{9}$ & $0(0,0 \%)$ & $4(1,0 \%)$ & $25(6,3 \%)$ & $225(56,8 \%)$ & $127(32,1 \%)$ & $15(3,8 \%)$ & $4,3 \pm 0,6$ \\
\hline
\end{tabular}

Ítem 1 - "La organización del evento es adecuada"; Ítem 2 - "Los espacios del festival son seguros"; Ítem 3 - "La gestión logística es responsable"; 4 - "Existe buena accesibilidad al palco de los festivales"; 5 - "El paisaje del festival es bonito"; Ítem 6 - "Existe buena recepción de los fans"; Ítem 7 - "La zona de acampada es adecuada"; Ítem 8 - "La limpieza general del evento es buena"; Ítem 9 - "La evaluación general de evento es positiva".

Analizando los valores medios de evaluación cognitiva de los ítems, vemos que el ítem con mayor nivel de concordancia es el ítem 5 - el paisaje del festival es bonito- con una media de 4,8 (sobre 5) y una desviación de 0,5. En segundo y tercer lugar se sitúan los ítems 2 -los espacios del festival son seguros- y el ítem 9 - la evaluación general del evento es positiva- con medias de 4,4 y 4,3, respectivamente. Con todo ello se puede afirmar que ICFVPC es positiva.

\section{Imagen cognitiva del destino turístico de Viana do Castelo (ICDTVC)}

En lo que respecta a la ICDTVC se proponen un total de 5 ítems a valorar con una escala Likert de 5 puntos, siendo 1 la valoración más baja y 5 la más alta. Tal y como se observa en la Tabla 5, la evaluación cognitiva de Viana do Castelo es elevada, ya que los valores medios son todos superiores o prácticamente iguales a 4 , además de que la moda (valor más repetido) es 5 en todos los casos. Destaca el ítem 2 -los servicios tienen buena calidad-, donde el 53,9\% de los encuestados le dan una valoración de 5 , el ítem 4 -el paisaje es bello-(63,3\% le dan la puntuación más alta) y el ítem 7 -el destino está limpio- (50,4\% lo valoran con un 5). 
Tabla 5. ICDTVC- atributos do destino de Viana do Castelo

\begin{tabular}{|c|c|c|c|c|c|c|c|}
\hline & \multicolumn{7}{|c|}{ Dimensión I- Recursos Turísticos } \\
\hline Ítems & $\mathbf{1}$ & $\mathbf{2}$ & $\mathbf{3}$ & $\mathbf{4}$ & $\mathbf{5}$ & No responde & $\begin{array}{c}\text { Media } \pm \\
\text { Desviación }\end{array}$ \\
\hline Ítem 1 & $2(0,5 \%)$ & $6(1,5 \%)$ & $45(11,4 \%)$ & $165(41,8 \%)$ & $173(43,8 \%)$ & $4(1,0 \%)$ & $4,3 \pm 0,8$ \\
\hline Ítem 2 & $2(0,5 \%)$ & $13(3,3 \%)$ & $72(18,2 \%)$ & $92(23,3 \%)$ & $213(53,9 \%)$ & $3(0,8 \%)$ & $3,9 \pm 0,8$ \\
\hline Ítem 3 & $4(1,0 \%)$ & $18(4,6 \%)$ & $97(24,6 \%)$ & $113(28,6 \%)$ & $160(40,5 \%)$ & $3(0,8 \%)$ & $3,9 \pm 0,9$ \\
\hline Ítem 4 & $1(0,3 \%)$ & $5(1,3 \%)$ & $21(5,3 \%)$ & $113(28,6 \%)$ & $250(63,3 \%)$ & $5(1,3 \%)$ & $4,6 \pm 0,7$ \\
\hline Ítem 5 & $2(0,5 \%)$ & $20(5,1 \%)$ & $77(19,5 \%)$ & $127(32,2 \%)$ & $164(41,5 \%)$ & $5(1,3 \%)$ & $4,0 \pm 0,9$ \\
\hline & \multicolumn{7}{|c|}{ Dimensión II - Infraestructura y Gastronomía } \\
\hline Ítem 6 & $6(1,5 \%)$ & $23(5,8 \%)$ & $87(22,0 \%)$ & $120(30,4 \%)$ & $152(38,5 \%)$ & $7(1,8 \%)$ & $3,8 \pm 0,9$ \\
\hline Ítem 7 & $3(0,8 \%)$ & $22(5,6 \%)$ & $50(12,7 \%)$ & $116(29,4 \%)$ & $199(50,4 \%)$ & $5(1,3 \%)$ & $4,0 \pm 0,8$ \\
\hline Ítem 8 & $14(3,5 \%)$ & $52(13,2 \%)$ & $60(15,2 \%)$ & $128(32,4 \%)$ & $136(34,4 \%)$ & $5(1,3 \%)$ & $3,4 \pm 1,0$ \\
\hline Ítem 9 & $12(3,0 \%)$ & $26(6,6 \%)$ & $27(6,8 \%)$ & $52(13,2 \%)$ & $116(29,4 \%)$ & $162(41,0 \%)$ & $3,2 \pm 1,1$ \\
\hline \multicolumn{7}{|c|}{ Dimensión III - Confort y Factor Económico } \\
\hline Ítem 10 & $10(2,5 \%)$ & $20(5,1 \%)$ & $42(10,6 \%)$ & $105(26,6 \%)$ & $163(41,3 \%)$ & $55(13,9 \%)$ & $3,4 \pm 0,9$ \\
\hline Ítem 11 & $2(0,5 \%)$ & $5(1,3 \%)$ & $41(10,4 \%)$ & $150(38,0 \%)$ & $187(47,3 \%)$ & $10(2,5 \%)$ & $4,3 \pm 0,8$ \\
\hline Ítem 12 & $16(4,1 \%)$ & $36(9,1 \%)$ & $44(11,1 \%)$ & $110(27,8 \%)$ & $160(40,5 \%)$ & $29(7,3 \%)$ & $3,4 \pm 1,0$ \\
\hline Ítem 13 & $15(3,8 \%)$ & $42(10,6 \%)$ & $74(18,7 \%)$ & $105(26,6 \%)$ & $149(37,7 \%)$ & $10(2,5 \%)$ & $3,6 \pm 1,0$ \\
\hline & \multicolumn{7}{|c|}{ Dimensión IV - Comunicación e Información } \\
\hline Ítem 14 & $9(2,3 \%)$ & $31(7,8 \%)$ & $87(22,0 \%)$ & $93(23,5 \%)$ & $167(42,3 \%)$ & $8(2,0 \%)$ & $3,8 \pm 1,0$ \\
\hline Ítem 15 & $9(2,3 \%)$ & $23(5,8 \%)$ & $62(15,7 \%)$ & $116(29,4 \%)$ & $175(44,3 \%)$ & $10(2,5 \%)$ & $3,7 \pm 0,9$ \\
\hline
\end{tabular}

Ítem 1 - "La población es hospitalaria”; Ítem 2 - "Los servicios tienen buena calidad”; Ítem 3 - "Hay variedad de entretenimiento"; Ítem 4 - "El paisaje es bello"; Ítem 5 - "El destino tiene variedad de eventos"; Ítem 6 - "Existe diversidad gastronómica"; Ítem 7 - "El destino está limpio"; Ítem 8 - "El desplazamiento a puntos de interés es sencillo"; Ítem 9 - "Los servicios del aeropuerto son de calidad"; Ítem 10 - "Los alojamientos hoteleros tienen diversidad"; Ítem 11 - "El destino es seguro"; Ítem 12 - "Existe buena accesibilidad de transportes públicos y privados"; Ítem 13 - "Existe buena relación calidad/precio"; Ítem 14 - "Existe suficiente señalización para llegar al destino"; Ítem 15 - "Existe accesibilidad a la comunicación".

Se observa también que los ítems 10 -los alojamientos hoteleros tienen diversidad- y 9 -los servicios del aeropuerto son de calidad- muestran tasas de no respuesta representativas (13,9\% y $41 \%$ respectivamente), justificable ya que gran parte de la muestra se aloja en la zona de acampada y acude al destino por otros medios. Al analizar los valores medios se deduce que el ítem con mayor nivel de concordancia es el 4 -el paisaje es bello- con una media de 4,6 (sobre 5) y una desviación de 0,7. Le siguen en la misma medida los ítems 1 - la población es hospitalaria- y 11 -el destino es seguro- con una media 4,3. Se puede por tanto afirmar que la evaluación cognitiva del destino turístico Viana do Castelo es positiva por la belleza de su paisaje, la hospitalidad de su población y la seguridad del propio destino. Como puntos negativos resaltar el desplazamiento a los puntos de interés (valoración media de 3,4), la diversidad de sus alojamientos hoteleros $(3,4)$, la accesibilidad del transporte $(3,4)$ y, sobre todo, la calidad de los servicios del aeropuerto, ítem con peor valoración $(3,2)$.

En el sentido de confirmar la hipótesis planteada en esta investigación, si existía una relación positiva entre ICFVPC e ICDTVC se han creado 4 dimensiones: dimensión 1- "recursos turísticos"; dimensión 2- "infraestructura y gastronomía"; dimensión 3- dimensión "confort y factor económico" y dimensión 4- "comunicación e información". Se ha efectuado un análisis de los diagramas de dispersión y se verifica que las variables están positivamente correlacionadas 
-tal y como se observa tras aplicar el test de Correlación de Spearman- (Buglear, 2012) (ver Tabla 6). Se verifica que cuanto mayor es el nivel de concordancia de evaluación cognitiva del FVPC, mayor es el nivel de concordancia de evaluación cognitiva del destino turístico de Viana do Castelo en la dimensión 1. Lo mismo se puede deducir sobre las dimensiones 2, 3 y 4 (Gráficos 1, 2, 3 y 4).

Tabla 6. Test de Correlación ICFVPC e ICDTVC de las 4 dimensiones

\begin{tabular}{|c|c|}
\hline Correlaciones & Test de Correlación de Spearman \\
\hline ACFVPC $\rightarrow$ ACDTVC / Dimensión 1 & $\rho=0,789 ; n=392 ; p<0,001$ \\
\hline ACFVPC $\rightarrow$ ACDTVC / Dimensión 2 & $\rho=0,823 ; n=392 ; p<0,001$ \\
\hline ACFVPC $\rightarrow$ ACDTVC / Dimensión 3 & $\rho=0,741 ; n=392 ; p<0,001$ \\
\hline ACFVPC $\rightarrow$ ACDTVC / Dimensión 4 & $\rho=0,732 ; n=392 ; p<0,001$ \\
\hline
\end{tabular}

En el test de Spearman $(\rho=0,789 ; n=392 ; p<0,001)$, como $p<0,05$ se concluye que existe una correlación significativa entre ACFVPC en la dimensión 1 de ACDTVC. De forma idéntica, se obtiene la misma conclusión en relación a la dimensión 2 - "Infraestructura y gastronomía" ( $\rho=0,823 ; n=392 ; p<0,001)$, dimensión 3 - "Confort y factor económico" $(\rho=$ $0,741 ; n=392 ; p<0,001)$ y dimensión 4 - "Comunicación e información" ( $\rho=0,732 ; n=392$; $p<0,001)$. Tras lo expuesto, se confirma la hipótesis de partida: la ICFVPC afecta positivamente a la ICDTVC.

Gráfico 1 - Diagrama de dispersión en la evaluación cognitiva de DTVC en la dimensión 1 y evaluación cognitiva del FVPC

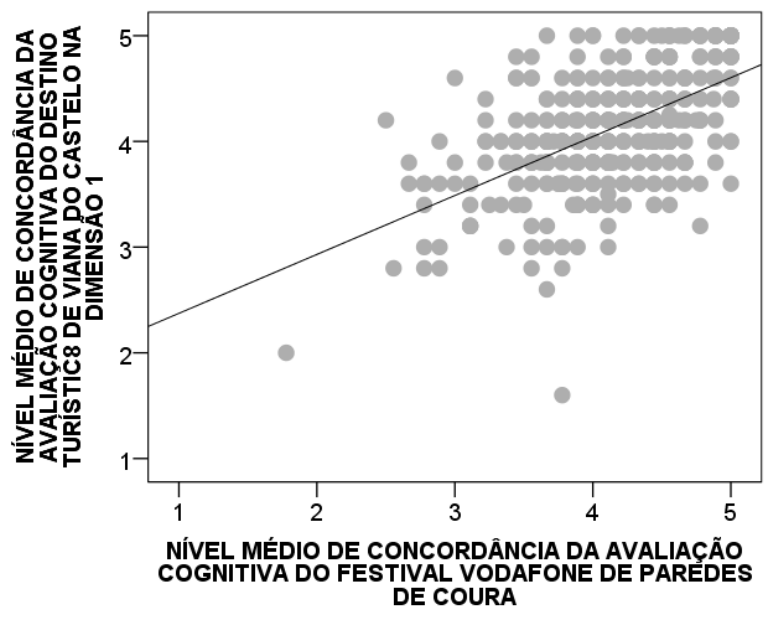

Gráfico 2 - Diagrama de dispersión en la evaluación cognitiva de DTVC en la dimensión 2 y evaluación cognitiva del FVPC

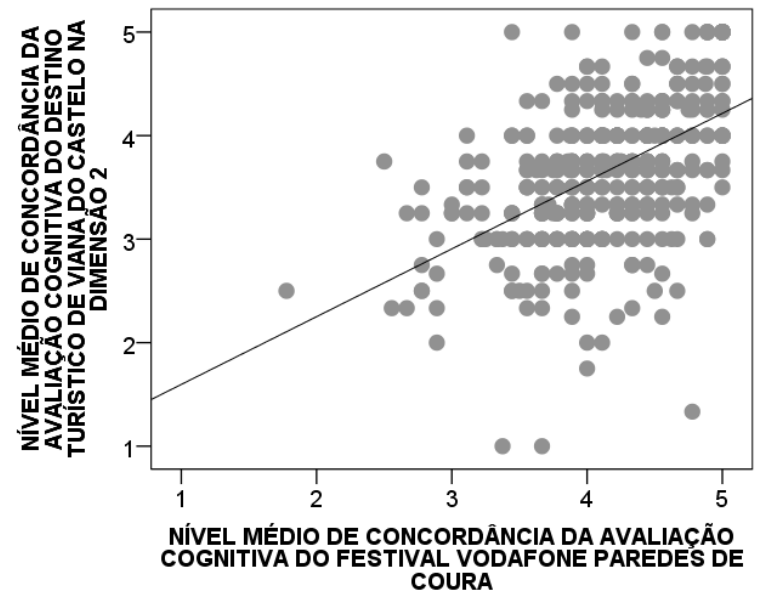


Gráfico 3 - Diagrama de dispersión en

la evaluación cognitiva de DTVC en la dimensión 3 y evaluación cognitiva del FVPC

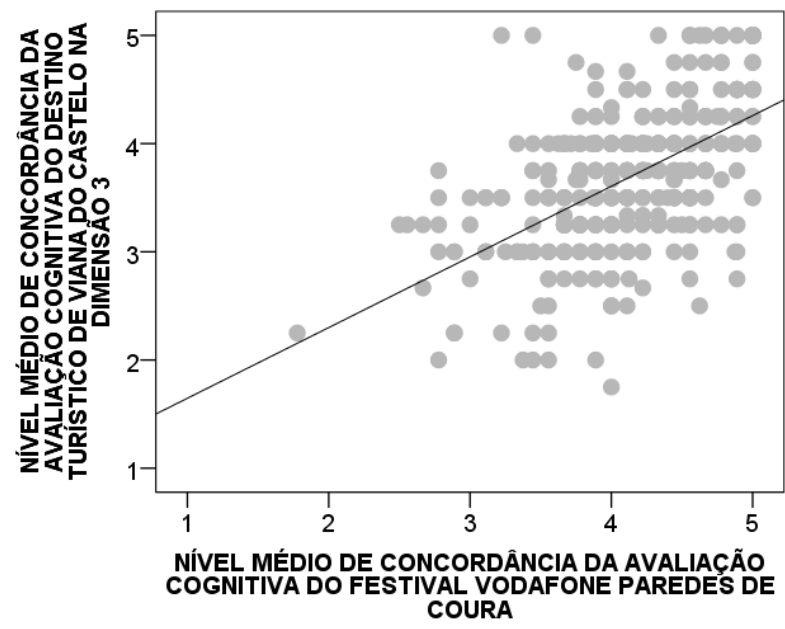

Gráfico 4 - Diagrama de dispersión en

la evaluación cognitiva de DTVC en la dimensión 4 y evaluación cognitiva del FVPC

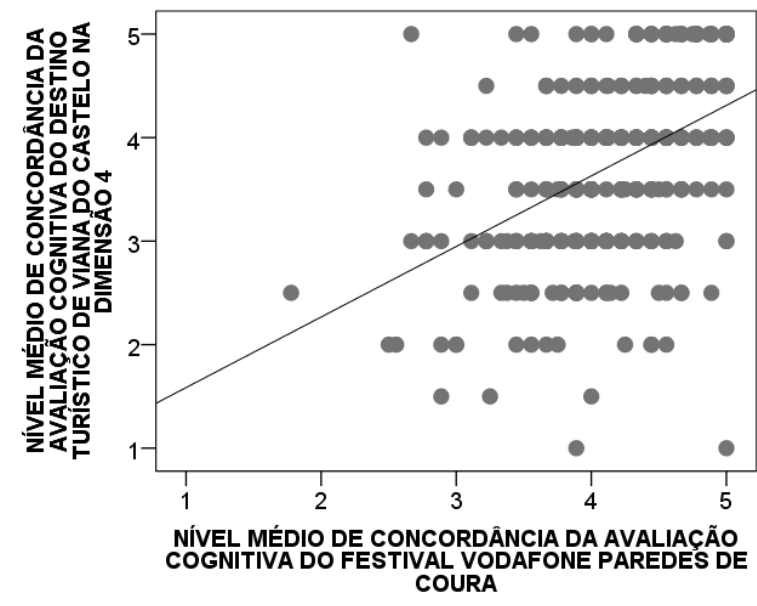

\section{CONCLUSIONES}

El cuidado y difusión de la imagen turística de un destino es clave a día de hoy ya que estudios previos han demostrado que influye de modo directo en la elección del destino y en la intención de revisita por parte del turista. Es por ello que los destinos turísticos buscan modos de enriquecer y ampliar su oferta turística de modo que esto repercuta en su imagen y en una mayor proyección de la misma. Entre los productos turísticos con un interés creciente se encuentran los megaeventos y eventos, difundiendo la imagen del destino a través de los mismos, ya que son muchos los individuos interesados en estos. Paralelamente a la visita al destino motivados por el evento, disfrutan del propio destino y conocen otros recursos.

Tras analizar el caso del Festival Vodafone Paredes de Coura se obtiene el perfil de asistente a este tipo de eventos (siendo la música alternativa la protagonista). Así, se establece un perfil de individuo joven, hombre o mujer y estudiante. Se caracteriza por asistir acompañado y como dato relevante motivado por el festival. Se demuestra así que el propio festival es el elemento atrayente de este tipo de turista. También se destaca entre los resultados obtenidos que la estancia más habitual son 5-7 días $(51,1 \%)$, mientras que en el festival la mayoría sólo permanece 4 (38\%). Este dato es muy positivo para el destino, ya que, aunque la mayoría de los asistentes acude a Viana do Castelo motivados por el festival, prolongan su estancia hasta 3 días más. En este caso la evaluación de la imagen cognitiva es tanto positiva para el festival como para el destino, pero las valoraciones son más altas para el propio destino. El festival presenta algunos aspectos mejorables, como la limpieza o el acceso a los palcos. Aun así, se demuestra que la imagen positiva del evento influye favorablemente en la imagen del destino, motivando al asistente a conocer también el destino una vez que asiste al festival.

Como implicaciones del estudio, y conforme a lo demostrado por los resultados del comportamiento del viaje, la mayoría de los participantes del festival permanece en el destino entre 4 y 7 días. Esto permite a los gestores del destino adoptar estrategias para rentabilizar la estancia en los alojamientos de la región. Además, la mayoría de los festivaleros son jóvenes, 
lo que permite adoptar estrategias de comunicación ajustadas a este segmento. A pesar de la contribución teórica y metodológica de este trabajo, éste presenta limitaciones. No fue posible evaluar la razón por la cual la mayoría de encuestados acampa en el propio recinto del festival.

Como futuras líneas de investigación se contemplará el estudio del perfil económico de los participantes de este tipo de festival para verificar si existe alguna relación económica o si solo se está ante una preferencia de alojamiento de un segmento joven. Matizar también que la imagen del destino es apenas un elemento de la marca del destino, por lo que futuras líneas de investigación deberán también contemplar el estudio de la relación de la marca del evento con la marca del destino.

Conforme a lo descrito en la revisión teórica, la imagen tiene dos componentes: cognitivo y afectivo. Este estudio se centra en el componente cognitivo, por lo que futuros trabajos podrán evaluar si el componente afectivo se comporta como el cognitivo.

Por último, los resultados confirman que la procedencia de los asistentes es portuguesa y española, por lo que se propone en futuras investigaciones el contemplar una pregunta para saber cuál es la fuente promocional a través de la cual han sabido del evento y analizar si las campañas efectuadas por los organizadores del evento son eficaces.

\section{VIII.REFERENCIAS BIBLIOGRÁFICAS}

Agapito, D., Valle, P. O., y Mendes, J. D. (2013). The Cognitive-Affective-Conative Model of Destination Image: A Confirmatory Analysis. Journal of Travel \& Tourism Marketing, 30 (5), 471-481. http://dx.doi.org/10.1080/10548408.2013.803393

Añaña, E. D., Anjos F., y Pereira, M. D. (2016). Imagem de Destinos Turísticos: avaliação à luz da teoria da experiência na economia baseada em serviços. Revista Brasileira de Pesquisa em Turismo, 10 (2), 309-329. http://dx.doi.org/10.7784/rbtur.v10i2.1093

Araújo Vila, N., y Domínguez Vila, T. (2012). Los festivales de cine como elemento potenciador de destinos turísticos. El caso de San Sebastián". Revista de Comunicación Vivat Academina, 121, 31-49.

APORFEST(AssociaçãoPortuguesa Festivais deMúsica)(2017).249festivais demúsicaem Portugal no ano de 2016. Online in http://www.aporfest.pt/single-post/2016/09/22/243-festivais-de-m\%C3\%BAsica-em-Portugal-no-ano-de-2016-estudo-Aporfest

Baloglu, S., y McCleary, K. W. (1999). A Model of Destination Image Formation. Annals of Tourism Research, 26 (4), 868-897 https://doi.org/10.1016/S0160-7383(99)00030-4

Beerli, A., y Martín, J. D. (2004). Tourists' characteristics and the perceived image of tourist destinations: a quantitative analysis-a case study of Lanzarote, Spain. Tourism Management, 25, 623-636 http://doi.org/10.1016/i.tourman.2003.06.004

Brea, J. F., y Cardoso, L. (2011). Tourism Destination Image: reflexão sobre as principais investigações internacionais. Cultur: Revista de Cultura e Turismo, 5(2), 4-18.

Buglear, J. (2012). Quantitative Methods for Business and Management. Harlow: Pearson.

Cardoso, L., y Brea, J. F. (2012). Medição da Tourism Destination Image: implicações para os markters do destino turístico. XVII Congresso AECIT (Associação Espanhola de Especialistas Científicos em Turismo), 1-16. 
Chierichetti, L. (2012). La promoción del turismo musical: Los festivales de música en Facebook. Pasos, Revista de Turismo y Patrimonio Cultural, 10 (4), 49-58.

Custódio, M. J. F., y Gouveia, P. M. (2007). Evaluation of the cognitive image of a country/ destination by the media during the coverage of mega-events: the case of UEFA EURO 2004TM in Portugal. International Journal of Tourism Research, 9(4), 285-296.

Echtner, C. M., y Ritchie, J. R. (2003). The Meaning and Measurement of Destination Image. Journal of Tourism Studies, 14(1), 37-48 https://doi.org/0.1177/004728759303100402

Estrada, M., Sotos, J., y Monteferrer, D. (2015). Los festivals musicales como experiencia turística: una aproximación teórica. International Journal of Marketing, Communication and New Media, 3(4), 43-53.

Gallarza, M. G., Saura, I. G., y García, H. C. (2002). Destination Image Towards a Conceptual Framework. Annals of Tourism Research, 29(1), 56-78 https://doi.org/10.1016/ $\underline{\text { S0160-7383(01)00031-7 }}$

Gartner, W. C. (1993). Image Formation Process. Journal of Travel \& Tourism Marketing, 2(2-3), 191-215 https://doi.org/10.1300/J073v02n02

Getz, D. (2008). Event tourism: definition, evolution, and research. Tourism Management, 29, 403-428. https://doi.org/10.1016/i.tourman.2007.07.017

Getz, D., y Page, S. J. (2016). Progress and prospects for event tourism research. Tourism Management, 52, 593-631.

Grappi, S., y Montanari, F. (2011). The role of social identification and hedonism in affecting tourist re-patronizing behaviours: the case of an Italian festival. Tourism Management, 32, 1128-1140. https://doi.org/10.1016/j.tourman.2010.10.001

Hallmann, K., Zehrer, A., y Müller, S. (2015). Perceived Destination Image: An Image Model for a Winter Sports Destination and Its Effect on Intention to Revisit. Journal of Travel Research, 54(1), 94-106 https://doi.org/10.1177/0047287513513161

Hosany, S., Ekinci, Y., y Uysal, M. (2007). Destination Image and Destionation Personality. International Journal of Culture, Tourism and Hospitality Research, 1(1), $62-81$ https:// doi.org/10.1108/17506180710729619

Hosany, S., y Witman, M. (2010). Dimensions of cruisers' experiences, satisfaction, and intention to recommend. Journal of Travel Research, 49, 351-364. https://doi. org/10.1177/0047287509346859

Hunter, W. C. (2016). The social construction of tourism online destination image: A comparative semiotic analysis of the visual representation of Seoul. Tourism Management, 54, 221-229 https://doi.org/10.1016/i.tourman.2015.11.012

Josiassen, A., Assaf, A. G., Woo, L., y Kock, F. (2016). The Imagery-Image Duality Model: An Integrative Review and Advocating for Improved Delimitation of Concepts. Journal of Travel Research, 55(6), 789-803.

Lee, J. S., Lee, C. K., y Choi, Y. (2011) Examining the role of emotional and functional values in festival evaluation. Journal of Travel Research, 50(6), 685-696. https://doi. org/10.1177/0047287510385465

Leenders, M. A. (2010). The relative importance of the brand of music festivals: a customer equity perspective. Journal of Strategic Marketing, 18(4), 291-301. 
Liu, Y. (2014). Cultural events and cultural tourism development: lessons from the European Capitals of Culture. European Planning Studies, 22 (3), 498-514. http://dx.doi.org/10.1 $\underline{080 / 09654313.2012 .752442}$

Llodrá-Riera, I., Martinez-Ruiz, M. P., Jiménez-Zarco, A. I., y Izquierdo-Yusta, A. (2015). A multidimensional analysis of the information sources construct and its relevance for destination image formation. Tourism Management, 48, 319-328 https://doi. org/10.1016/j.tourman.2014.11.012

Llopis Amorós, M. P., Gil Saura, I., y Ruiz Molina, M. E. (2017). Comunicaciones de marketing y valor de marca de un festival musical.

Loureiro, S. C., y Araujo, A. B. (2015). Negative Film Plot and Tourists' Image and Intentions: The Case of City of God. Journal of Travel \& Tourism Marketing, 32(4), 352-365 https:// doi.org/10.1080/10548408.2014.896769

Mak, A. H. (2017). Online destination image: Comparing national tourism organisation's and tourists' perspectives. Tourism Management, 60, 280-297 https://doi.org/10.1016/i. tourman.2016.12.012

Martín-Santana, J. D., Beerli-Palacio, A., y Nazzareno, P. A. (2017). Antecedents and consequences of destination image gap. Annals of Tourism Research, 62, 13-25 https://doi. org/10.1016/i.annals.2016.11.001

Morales, M. J., y Vela, J. D. (2009). Identidad territorial y promoción turística : la organización de eventos como estrategia de creación, consolidación y difusión de la imagen de marca del territorio. Zer - Revista de Estudios de Comunicación, 14(26), 277-297.

Önder, I., y Marchiori, E. (2017). A comparison of pre-visit beliefs and projected visual images of destinations. Tourism Management Perspectives, 21, 42-53.

Paiva, R. (2015). Eventos e megaeventos: ócio e negócio no turismo. Revista Brasileira de Pesquisa em Turismo, 9(3), 479-499.

Pan, B., y Li, X. (2011). The long tail of destination image and online marketing. Annals of Tourism Research, 38(1), 132-152 https://doi.org/10.1016/j.annals.2010.06.004

Pavluković, V., Armenski, T., y Alcántara-Pilar, J. (2017). Social impacts of music festivals: Does culture impact locals' attitude toward events in Serbia and Hungary? Tourism Management, 63, 42-53.

Pike, S. (2016). Destination image: Identifying baseline perceptions of Brazil, Argentina and Chile in the nascent Australian long haul travel market. Journal of Destination Marketing \& Management, 5, 164-170 https://doi.org/10.1016/i.jdmm.2015.12.006

Prat, J. M. (2014). Las relaciones sociales y las motivaciones para asistir al festival de música de peralada. Boletín de la Asociación de Geógrafos Españoles, 66, 207-221.

Santos, J. D., Vareiro, L., Remoaldo, P., y Ribeiro, J. C. (2017). Cultural mega-events and the enhancement of a city's image : differences between engaged participants and attendees. Journal of Policy Research in Tourism, Leisure \& Events, 9(2), 129-151 http://doi. org/10.1080/19407963.2016.1157598

Seonjeong, A. M. Liang, L. R., y Chiang, T. L. (2014). The experience economy approach to festival marketing: Vivid memory and attendee loyalty. Journal of Services Marketing, 28 (1), 22-35. https://doi.org/10.1108/JSM-06-2012-0105

Stepchenkova, S., y Li, X. (2014). Destination image: Do top-of-mind associations say it all? Annals of Tourism Research, 45, 46-62 https://doi.org/10.1016/i.annals.2013.12.004 
Stepchenkova, S., y Morrison, A. M. (2008). Russia's destination image among American pleasure travelers: Revisiting Echtner and Ritchie. Tourism Management, 29(3), 548-560 https://doi.org/10.1016/j.tourman.2007.06.003

Stylos, N., Bellou, V., Andronikidis, A., y Vassiliadis, C. A. (2017). Linking the dots among destination images, place attachment, and revisit intentions: A study among British and Russian tourists. Tourism Management, 60, 15-29 https://doi.org/10.1016/j. tourman.2016.11.006

Tanford, S., y Jung, S. (2017). Festival attributes and perceptions: A meta-analysis of relationships with satisfaction and loyalty. Tourism Management, 61, 209-220.

Weaver, D. B., y Lawton, L. J. (2013). Resident perceptions of a contentious tourism event. Tourism Management, 37, 165-175.

Yan, G., Kloeppel, M., y Li, X. (2017). Producing Extreme Metal festivals: An analysis from Lacan's gaze. Tourism Management, 59, 579-589 https://doi.org/10.1016/i. tourman.2016.09.014

Zucco, F. D., de Quadros, C. M., Schmitt, J., y Fiuza, T. F. (2017). Imagem e identidade turísticas relacionadas às práticas e bens culturais percebidas pelos residentes: perspectivas a partir da cidade de Blumenau, Santa Catarina, Brasil. Revista Brasileira de Pesquisa em Turismo, 11(2), 320-346. 